\title{
ChK1 Activation Induces Reactive Astrogliosis Through CIP2A/PP2A/STAT3 Pathway In Alzheimer's Disease
}

\section{Ying Zhou}

Department of Nephrology,The First Affliliated Hospital of Wenzhou Medical University

Xiaoyuan Liu

Wenzhou Medical University

Shuqing Ma

Wenzhou Medical University

Dichen Yang

Wenzhou Medical University

Nan Zhang

Wenzhou Medical University

Simin Ye

Wenzhou Medical University

Qiongying Zhang

Department of Pathology,The First Affiliated Hosptital of Wenzhou Medical University

Jing Ruan

Department of Pathology, The First Affiliated of Wenzhou Medical University

Jun Ma

Department of Pathology, The First Affiliated Hospital of Wenzhou Medical University

\section{Shiyi Wang}

Wenzhou Medical University

\section{Nan Jiang}

Wenzhou Medical University

\section{Zongyuan Zhao}

Wenzhou Medical University

\section{Shujue Zhao}

Wenzhou Medical University

Chenfei Zheng

Wenzhou Medical University First Affiliated Hospital Department of Hematology

\section{Xiaofang Fan}

Institute of Hypoxia,School of Basic Medical Science,Wenzhou Medical University

\section{Yongsheng Gong}


Institute of Hypoxia Medicine,School of Basic Medcal Sciences,Wenzhou Medical University

\section{Xiaochuan Wang}

Institute of Pathophysiology,Key Laboratory of Ministry of Education for Neurological

Disorders,Huazhong University of Science and Technology

\section{Junming Fan}

Institute of Hypoxia Medicine,School of Basic Medical Sciences,Wenzhou Medical University

\section{Jianmin Li}

Department of Pathology,The First of Affiliated Hospital of Wenzhou Medical University

\section{Rong Liu}

Technology and Science Institute of Northern Taiwan: Taipei City University of Science and Technology Yangping Shentu ( $\nabla$ styp@wmu.edu.cn )

The First Affiliated Hospital of Wenzhou Medical University

\section{Research}

Keywords: ChK1, STAT3, CIP2A, Astrogliosis, Alzheimer disease

Posted Date: September 20th, 2021

DOl: https://doi.org/10.21203/rs.3.rs-877963/v1

License: (c) (1) This work is licensed under a Creative Commons Attribution 4.0 International License. Read Full License

Version of Record: A version of this preprint was published at The FASEB Journal on February 23rd, 2022. See the published version at https://doi.org/10.1096/fj.202101625R. 


\section{Abstract}

Background: In Alzheimer's disease (AD), activation of astrocyte participates in the development of neurodegenerative diseases through neuroinflammation and disturbs glia-neuron interaction. Cancerous Inhibitor of PP2A (CIP2A) is an endogenous PP2A inhibitor. CIP2A upregulation specifically in astrocytes causes reactive astrogliosis, synaptic degeneration and cognitive deficits. However, the underlying mechanism of CIP2A upregulation remains unclear.

Methods: In 3xTg-AD mice, we determined ChK1 was activated and related to DNA damage upregulating CIP2A by WB. We transfected EGFP-ChK1 plasmid into HEK293-T cell to determine ChK1 induces CIP2A upregulation and PP2A inhibition. We incubated $A B$ and infected GFAP-ChK1-LV into primary astrocytes to confirm the signaling pathway in astrocytes and astrogliosis in AD. GFAP-ChK1-AAV was injected into C57/BL6 mice to induce specific expression of target protein in astrocytes. ChK1 inhibitor (SB) was performed to reverse the ChK1 activity. Outcomes were assessed using molecular (immunofluorescent staining, Western Blot and Golgi staining) measures to estimate symptomatic pathology and behavioral (NORT, OLT, MWM and FCT) measures to assess cognitive function. For most experiments, subjects were randomly assigned to experimental groups, and data were collected under blinded experimental conditions.

Results: We demonstrated that DNA damage related Checkpoint kinase 1 (ChK1) was activated in 3xTgAD mice. ChK1-mediated CIP2A overexpression drove inhibition of PP2A and activated STAT3, then led to reactive astrogliosis and neurodegeneration in vitro. Infection of mouse brain with GFAP-ChK1-AAV induced $A D$-like cognitive deficits and exacerbated $A D$ pathologies in vivo. In conclusion, we showed that ChK1 activation induced reactive astrogliosis, degeneration of neurons and deterioration of AD through CIP2A-PP2A-STAT3 pathway, and inhibiting ChK1 might be a potential therapeutic approach for AD treatment.

Conclusions: These results suggest that ChK1 is upregulated in 3XTg-AD mice, ChK1-mediated CIP2A overexpression drives inhibition of PP2A and activates STAT3, then leads to reactive astrogliosis, neurodegeneration and $\mathrm{AD}$-like cognitive deficits in vitro and in vivo.

\section{Background}

Alzheimer's disease (AD) is a common neurodegenerative disease with underlying mechanism unclarified. The pathological characters of $A D$ are $A B$ deposition,tau hyperphosphorylation, neurodegeneration, as well as reactive gliosis [1,2]. Glia in cell number are five to ten times of neurons, increasing body of studies have showed that abnormal activation of glia participated in the development of neurodegenerative diseases through neuroinflammation and disturbed glia-neuron interaction $[3,4]$. Astrocyte as the major type of glia cell also plays a crucial part in neurodegenerative diseases. Studies have reported that in the early stage of $A D$, activation of astrocyte would lead to loss of neurons and 
synapses, and accumulation of pathogenic proteins [5, 6]. However, the mechanisms underlying the activation of astrocytes in AD have not been completely understood.

Cancerous inhibitor of PP2A (CIP2A) is an endogenous inhibitor of Protein Phosphatase 2A (PP2A) [7-9]. PP2A is the most important tau phosphatase. In AD brains, PP2A activity is decreased $[10,11]$. We reported that in AD brains, CIP2A is upregulated. Increased CIP2A levels correlates to PP2A inhibition, and CIP2A overexpression in neurons induces hyperphosphorylation of PP2A substrates tau and APP, neurodegeneration and cognitive deficits in mice [12]. Furthermore, CIP2A upregulation specifically in astrocytes can induce reactive astrogliosis, the latter, promotes synaptic impairments both in cells and mouse brains [13]. As an oncoprotein, expression of CIP2A is upregulated by activation of Checkpoint kinase 1 (ChK1) upon DNA damage in tumor cells [14]. Whether ChK1 activation also participate in CIP2A upregulation and astrocytes activation in $A D$ remains unanswered.

STAT3 (Signal transducer and activator of transcription 3) as a signal transducer is associated with many biological processes including cell survival, differentiation, growth and immune response [15]. PP2A inactivation stimulates STAT3, leading to proliferation of tumor cells [16-18]. These findings indicate that STAT3 activation can be induced by PP2A inhibition, and promotes cell proliferation. In AD human and transgenic mouse brains, STAT3 is activated. Recently, Reichenbach et al showed that in STAT3 deleted 3XTg-AD mice, astrocytes had better ability to internalize A $\beta$ and produced less inflammatory cytokines [19], indicating a role of STAT3 in astrogliosis in AD brain.

Taking all these events together, we suspect that ChK1-CIP2A-PP2A-STAT3 signaling is activated in astrocytes in AD brains and participate in AD-like astrogliosis. In the present study, we aimed to investigate the change of ChK1-CIP2A-PP2A-STAT3 signaling pathway in 3XTg-AD mice, and further identified the hypothesis both in cell and animal models.

\section{Material And Methods}

\section{Antibodies and reagents}

All primary antibodies used in this study were as follow: $\mathrm{YH} 2 \mathrm{~A} . \mathrm{X}$ (Proteintech, Cat\#10856-1-ap), S345 (Gene Tex, Cat\#39233), ChK1 (Bioss, Cat ac01184523; Abclonal, Cat\#a7653), S727-STAT3 (Affinity, Cat\#af3294), STAT3 (Zenbio, Cat\#385805), CIP2A (Cell Signaling Technology, Cat\#14805), GluA1 (Cell Signaling Technology, Cat\#13185), Synaptophysin (Abcam, Cat\#ab32127), ß-actin (Abcam, Cat\#ab8226), Synapsin 1 (Millipore, Cat\#S193), GAPDH (Proteintech, Cat\#60004-1-lg).

Chk1 plasmid and GFAP-ChK1-AAV were from Shanghai Genechem Co.,Ltd. (Shanghai, China). Neofect DNA transfection reagent was purchased from Neofect Biological Technology Co, Ltd (Beijing, China). Neurobasal, B27, DMEM-high and protein marker were from Invitrogen (Grand Island, NY, USA). DMEM/F12 and fetal bovine serum (FBS) were purchased from GIBCO (Grand Island, New York, USA). Serine/Threonine Phosphatase Assay kit was purchased from Promega Company (Madison, Wisconsin, USA). Human or rat or mouse A $\beta 40 / A \beta 42$ ELISA kits were purchased from Elabscience Biotechnology 
(Wuhan, China). Nissl staining solution was from Beyotime biotechnology (Shanghai, China). Golgi staining solution was from GENMED SCIENTIFICS INC. USA.

\section{Animals and AAV delivering}

For animal experiments, Sprague-Dawley rats and C57BL/6J mice were from Zhejiang Vital river Experimental Animal Technology Co. LTD Tongxiang branch. 3xTg-AD mice carrying human mutated APP, PS1 and tau genes were from Jackson Lab. All the mice can take food and water freely in an airconditioned room $\left(22 \pm 2^{\circ} \mathrm{C}, 12\right.$ hours light/dark cycle). The behavior tests were performed on their active hours.

For AAV injection, C57 mice were deeply anesthetized with isoflurane. AAV virus particles $(2.0 \mu \mathrm{l}$ at 0.4 $\mu \mathrm{l} / \mathrm{min}$ ) was injected into the lateral ventricle (interaural $3.58 \mathrm{~mm}$, bregma $0.22 \mathrm{~mm}$, depth $0.30 \mathrm{~mm}$ ). After the injection, the mice were kept under standard laboratory conditions.

\section{HEK 293-T cell culture and transfection}

For HEK293-T cell culture, the cells were cultured in DMEM-high medium supplemented with $10 \%$ FBS, $100 \mathrm{U} / \mathrm{mL}$ penicillin, and $0.1 \mathrm{mg} / \mathrm{mL}$ streptomycin at $37^{\circ} \mathrm{C}$ in the presence of $5 \% \mathrm{CO}_{2}$. The cells were cultured to $50 \%-60 \%$ confluence in 6 -well plates and changed to fresh medium, then transfected with relevant plasmids together with Neofect DNA transfection reagent (Neofect Biological Technology, Beijing, China). After 48 hours, cells and culture media were collected for further detections.

\section{Primary astrocyte and neuron culture}

Primary astrocyte culture and neuron culture were performed following the method described previously $[13,20]$. At the end of treatments, cells were collected and lyzed in RIPA buffer for further biological detections or fixed with $4 \%$ paraformaldehyde for immunofluorescence imaging.

\section{Western blotting and Co-IP}

For Western blotting, brain tissue homogenates or cell lysates were boiled at $100^{\circ} \mathrm{C}$ for $5 \mathrm{~min}$ in the loading buffer (50 mM Tris-HCl, pH 7.6, 2\% SDS, 10\% glycerol, $10 \mathrm{mM} \mathrm{DTT}$, and $0.2 \%$ bromphenol blue). The proteins were electrophoresed in 10\% SDS-polyacrylamide gel and the separated proteins were transferred to nitrocellulose membranes (Amersham Biosciences). The membranes were then blocked with $5 \%$ nonfat milk dissolved in TBS-Tween-20 (50 mM Tris- $\mathrm{HCl}, \mathrm{pH} 7.6,150 \mathrm{mM} \mathrm{NaCl}, 0.2 \%$ Tween-20) for $1 \mathrm{~h}$ and probed with primary antibody at $4^{\circ} \mathrm{C}$ overnight. Then the blots were detected using secondary antibodies at room temperature for $1 \mathrm{~h}$ and visualized using the Odyssey Infrared Imaging System (LicorBiosciences, Lincoln, NE, USA). The protein bands were quantitatively analyzed by Image $\mathrm{J}$ software (Rawak Software, Inc. Germany).

To analyze protein-protein interactions, Co-IP experiments were performed. Cells were lysed using Pierce IP lysis buffer (Thermo Fisher Scientific) supplemented with protease inhibitor cocktail (Roche). The cell 
lysates were centrifuged, and then, immunoprecipitated overnight at $4^{\circ} \mathrm{C}$ using the indicated primary antibodies followed by incubation with Dynabeads Protein G (Life Technologies) for $1 \mathrm{~h}$ The immunocomplexes were washed twice with IP lysis buffer before being resolved by SDS-polyacrylamide gel electrophoresis (SDS-PAGE) and immunoblotted with indicated antibodies.

\section{Fluorescence imaging and confocal microscopy}

For cultured neurons, cells were fixed with $4 \%$ paraformaldehyde for $15 \mathrm{~min}$, permeabilized in $0.5 \%$ Triton X-100 for $10 \mathrm{~min}$, followed by incubation with $3 \%$ bovine serum albumin (BSA) to block nonspecific sites. For mouse brain slices, sectioned slices were incubated with $3 \%$ bovine serum albumin (BSA) to block nonspecific sites. After blocking, primary antibody incubation was performed overnight at $4^{\circ} \mathrm{C}$. Alexa $488-$ conjugated secondary antibody (1:200, A-21206, Invitrogen) was used for fluorescence labeling. All the images were observed under the LSM710 confocal microscope (Zeiss, Germany).

For cultured astrocytes, the previous steps were as above. After blocking, primary antibodies incubation was performed overnight at $4^{\circ} \mathrm{C}$. Alexa 488 and 543-conjugated secondary antibody (1:200, A-21206, A10036, Invitrogen) were used for double fluorescence labeling. All the images were observed under the LSM710 confocal microscope (Zeiss, Germany).

\section{ChK1 and PP2A activity and ELISA assay}

ChK1 and PP2A activity was measured according to the protocols provided by the manufacturers (ChK1, GMS50155.2 GENMEDSCIENTIFICS INC. U.S.A; PP2A, V2460 kit; Promega). The culture medium or brain tissue homogenates which were lyzed in RIPA buffer were collected. The levels of IL-6, TNF-a, AB40 and $A \beta 42$ were detected by ELISA following the construction offered by the assay kit manufacturer (Elabscience Biotechnology, Wuhan, China).

\section{Behavior Tests}

\section{Novel objective recognition test]NORT!}

The mice were habituated to the arenas $(50 \mathrm{~cm} \times 50 \mathrm{~cm} \times 50 \mathrm{~cm}$ wooden container which was from Techman Software Co., Ltd., Chengdu, China) for 5 min without objects $24 \mathrm{~h}$ prior to the test. Arenas were cleaned with $70 \%$ ethanol between each habituation period. The day after the mice re-entered the arenas from the same starting point and were granted 5 minutes to familiarize themselves with the $A$ object and B object. One hour after the familiarization period, B object was replaced with $\mathrm{C}$ object, and the mice were granted 5 minutes to explore both objects. After $24 \mathrm{~h}, \mathrm{C}$ object was replaced with D object, and the mice were granted 5 minutes to explore both objects. All the behavior was recorded by a camera above the arena. The recognition index was calculated by $T A /(T A+T B), T B /(T A+T B), T C /(T A+T C), T D /(T A+T D)$. The discrimination index was calculated by (TC-TA)/(TA+TC), (TD-TA)/(TA+TD). TA, TB, TC, TD were respectively the time mice exploring the object $A, B, C$ and $D$.

\section{Object location testnOLT0}


The animals were habituated to the container that is same as novel objective recognition test. During the training session at the 6th day, the mice freely explored the floor of the box that contained two different objects. To get a measure of the OLT, one object was moved $45 \mathrm{~cm}$ to a new position, the total time of exploration of the familiar and novel object localization was measured 24 and 48 hours after the training. The objects and boxes were cleaned with ethanol after every training or test.

\section{Morris water maze test}

Spatial learning and memory were detected by Morris Water Maze (Techman Software Co., Ltd., Chengdu, China). Briefly, a circular arena $(120 \mathrm{~cm} \times 50 \mathrm{~cm})$ was filled with water $\left(23 \pm 2{ }^{\circ} \mathrm{C}\right)$. An escape platform $(10 \times 10 \times 15 \mathrm{~cm})$ was placed into the pool, $1.5 \mathrm{~cm}$ below the water surface. The water was made opaque by the addition of a white titanium dioxide. The test room contained several permanent extra-maze cues such as posters, a flag or other objects on the walls. A video-tracking camera above the center of the pool surface monitored the trajectory of the mice. Learning consisted of six consecutive daily acquisition sessions, each of them consisting of four trials, with a maximum trial duration of $60 \mathrm{~s}$. Latency time (s) to find the hidden platform were recorded during each trial of each learning session. If the mice found the platform within the maximum trial time allowed, it was left on the platform for $20 \mathrm{~s}$. If the mice did not find the platform within the time limit, it was gently placed on the platform for a $20 \mathrm{~s}$ period. Probe tests were carried out 1 or 2 days after acquisition.

\section{Fear conditioning test}

The fear conditioning test paradigm was performed following the methods previously described. In brief, the test was conducted in a conditioning chamber $(33 \mathrm{~cm} \star 33 \mathrm{~cm} \star 33 \mathrm{~cm})$ equipped with white board walls, a transparent front door, a speaker, and a grid floor (Techman Software Co., Ltd., Chengdu, China). On day 1 , mice were placed into the conditioning chamber and allowed free exploration for 2 minutes before the delivery of the conditioned stimulus (CS) tone (20 seconds, $80 \mathrm{~dB}, 2000 \mathrm{~Hz}$ ) paired with a foot shock unconditioned stimulus (US; 2 seconds, $0.95 \mathrm{~mA}$ ) through a grid floor at the end of the tone. A total of five CS-US pairs with a 60-s intertrial interval (ITI) were presented to each animal in the training stage. The mouse was removed from the chamber 1 minute after the last foot shock and placed back in its home cage. The contextual fear conditioning stage started 24 hours after the training phase, when the animal was put back inside the conditioning chamber for 5 minutes. The animal's freezing responses to the environmental context were recorded. The animal was placed back into the same chamber with different contextual cues, including blue wall, smooth plastic floor, and alcohol drops condition for 5 minutes, and the animal's freezing responses to the altered context were recorded. The tone fear conditioning stage started 24 hours after the different contextual stage. After 2 minutes of free exploration, the mouse was exposed to the exact same 3-CStones with 20-s ITI in the training stage without the foot shock, and its freezing responses to the tones were recorded.

\section{Nissl staining}


Thirty micrometers of coronal sections were mounted on gelatin-coated slides. Then, the sections were incubated in Cresyl violet for 3 minutes at room temperature, following with dehydration through $50 \%$, $75 \%, 95 \%$, and $100 \%$ alcohol, cleaning in xylene, and cover slipped with neutral balsam. Images were observed using light microscope.

\section{Golgi staining}

The mice were anesthetized and perfused intracardially with $400 \mathrm{ml}$ normal saline containing $0.5 \%$ sodium nitrite, followed with $400 \mathrm{ml} 4 \%$ formaldehyde and the Golgi dye solution containing $5 \%$ chloral hydrate, $4 \%$ formaldehyde, and $5 \%$ potassium dichromate. After perfusion, the brains were dissected into $5 \mathrm{~mm} \times 5 \mathrm{~mm}$ sections and transferred to a vial containing Golgi dye solution for 3 days in dark, then immersed in solution containing $1 \%$ silver nitrate for another 3 days. The brains were serially sectioned into $100 \mu \mathrm{m}$ thick slices using a vibrating microtome (Leica, VT1000S, Germany). Images were observed under the microscope (Nikon, Tokyo, Japan).

\section{Statistical analysis}

Data are expressed as mean \pm SEM and analyzed using GraphPad Prism 8 statistical software (USA, GraphPad Software). The one-way analysis of variance (ANOVA) procedure followed by Tukey was used to determine the differences among groups. For the comparison between two groups, student-t test was used. The significance was set at $P<0.05$. All results shown correspond to individual representative experiments.

\section{Results}

\section{Increased DNA damage, ChK1 activation and CIP2A upregulation in 3xTg-AD mice brains}

To explore the role of ChK1 in astrogliosis in AD, we first examined whether ChK1 is activated in the brains of 3xTg-AD mice. Compared to the control, DNA damage marker $\mathrm{YH} 2 \mathrm{~A}$.X, active form of ChK1 (ChK1 phosphorylated at S345), and CIP2A levels were all increased in 3xTg-AD mouse brains (Fig. 1A, B). Of note, after linear fitting, we found that $\mathrm{YH} 2 \mathrm{~A}$.X and ChK1-S345 were highly correlated (Fig. 1C). ChK1-S345 and CIP2A correlation achieved similar result (Fig. 1D). These data suggest that in AD brains, ChK1 activation due to DNA damage may upregulate CIP2A. We further identified the upregulation of CIP2A and the following PP2A inhibition upon ChK1 activation in HEK293-T cells overexpressed with ChK1 plasmid (Supplemental figure 1).

\section{A $\beta$ induces DNA damage, ChK1-CIP2A-PP2A-STAT3 signaling axis activation and astrogliosis in primary astrocytes}

To further confirm the signaling transduction from ChK1 to STAT3 in astrocytes and astrogliosis in AD, we detected the protein molecules in the signaling axis in primary astrocytes treated with $A \beta$, with or without pre-incubation of ChK1 inhibitor SB. A $B$ incubation increased $\gamma \mathrm{H} 2 \mathrm{~A}$.X, ChK1-S345, CIP2A, the active form of STAT3 (S727 phosphorylated STAT3) and GFAP levels, and SB treatment reversed ChK1 
activation, CIP2A overexpression, STAT3 activation and astrogliosis induced by A (Fig. 2A, B). We also found that the expression level of GFAP highly correlated with ChK1-S345, indicating ChK1 activation promotes astrogliosis (Fig. 2C). ChK1 activation and PP2A inhibition by A $\beta$ was confirmed by ChK1/PP2A activity assay results. Again, SB treatment partially reversed these changes (Fig. 2D, E). According to previous studies, activated astrocytes can release toxic factors to induce neuronal damage and death [21]. To identify that neurotoxic factors released from $A \beta$-activated astrocytes also promote neurodegeneration, we incubated rat primary neurons (DIV 8) with conditioned medium from $A \beta$-treated astrocytes $(A \beta-A C M)$ or $A \beta+S B$-treated astrocytes $(A \beta+S B-A C M)$ for 4 days. Synaptic proteins including Syn, Syn I, PSD95, and GluA1 were all decreased upon AB-ACM incubation, while ACM from SB+A treated astrocytes showed less toxicity to neurons (Supplemental figure 2A, B). Therefore, activated ChK1 which is induced by $A \beta$ probably promotes the proliferation and activation of astrocytes through CIP2APP2A-STAT3 signaling pathway, leading to neuronal degeneration.

\section{Overexpression of ChK1 induces CIP2A-PP2A-STAT3 signaling axis activation and reactive astrogliosis in primary astrocytes}

To further identify the hypothesis that ChK1 promotes astrogliosis through activating STAT3, we overexpressed ChK1 through Lenti-virus infection with GFAP promoter in primary astrocytes. Inflorescence imaging of astrocytes with anti-GFAP illustrated ChK1 motivated reactive astrogliosis characterized by hypertrophic cell bodies and more processes in ChK1-overexpressed astrocytes. SB prevented the reactive astrogliosis induced by ChK1 overexpression (Fig. 3A). We revealed the interaction of CIP2A, STAT3, and ChK1 through coimmunoprecipitation (Fig. 3B). Western blotting showed that overexpression of ChK1 increased CIP2A, S727-STAT3 and GFAP protein levels, which indicating the activation of astrocytes. But in the group of SB treatment, CIP2A-STAT3 signaling activation and reactive astrogliosis were prevented (Fig. 3C, D). We also found that the expression level of CIP2A protein raised along with the ChK1 levels (Fig. 3E). It has been shown that reactive astrocytes may acquire toxicity by releasing pro-inflammatory cytokines [22]. We thus measured the release of cytokines from ChK1overexpressed astrocytes. The results showed that cytokines like IL-6 and TNF- $a$ in the culture media increased significantly in ChK1 group compared with control group, and SB effectively reduced the cytokine levels (Fig. 3F). Reactive astrocytes can also produce and release considerable levels of $A \beta[23,24]$. Thus, we measured the $A \beta$ levels in the culture medium. Both $A \beta_{40}$ and $A \beta_{42}$ levels were dramatically increased in ChK1 group, which could be reversed by ChK1 inhibitor SB (Fig. 3G). In summary, these data indicate that overexpressed ChK1 causes reactive astrogliosis, accompanied by secretion of inflammatory cytokines and A 3 through ChK1-CIP2A-STAT3 signaling pathway.

\section{ChK1-ACM induces neurodegeneration}

To figure out whether neurotoxic factors released from ChK1-activated astrocytes also promote neurodegeneration, we incubated rat primary neurons (DIV 8) with conditioned medium from ChK1overexpressed astrocytes (ChK1-ACM) or ChK1+SB-treated astrocytes (ChK1+SB-ACM) for 4 days. At the end of incubation, after labeling neurons with MAP-2 antibody, we observed the decreased neurites which 
happened in the ChK1 group could also be reversed by SB (Fig. 4A). Western blotting analysis revealed a significant reduction in synaptic proteins including Syn, Syn I, PSD95, and GluA1 in the ChK1 group, but not in SB+ChK1 group (Fig. 4 B, C). In conclusion, these results indicate that ChK1-ACM can promote synaptic degeneration.

\section{Overexpression of ChK1 in astrocytes induces cognitive deficits in mice}

To further determine the role of ChK1 activation in astrocytes in AD, we explored the effect of specific ChK1 activation in astrocytes on cognition in animal models. GFAP-ChK1-AAV was injected into the lateral ventricle of C57/BL6 mice aged 8-10 weeks before behavioral tests and postmortem analysis (Fig. 5A, B). In order to induce specific expression of target protein in astrocytes, we constructed an AAV vector expressing ChK1 under the direct control of a human GFAP promoter (Fig. 5C). Four weeks after the virus infection, ChK1 overexpression in hippocampus was confirmed by immunofluorescence staining of brain slices (Fig. 5D). ORM and OLM tests were performed first in behavioral tests (Fig. 5E, F). Fourdays experiment was tailored for the mice in ORM, including one day for habitation, one day for training ( $A$ and $B$ ) and two days for test. After 24 and 48 hours retention interval, a novel object with a different shape and color ( $C$ or $D)$ was taken to replace the object $B$ for testing memory retention (Fig. 5E). There was no difference between the two groups in recognition index in the acquisition trial (Fig. 5G). As for the two tests, the control group favored the new object with comparable recognition and discrimination index, while ChK1 mice did not (Fig. 5H-J), indicating ORM is impaired in ChK1 mice. Subsequently, the same mice underwent an OLM task. To this end, the animals were habituated to an empty white box on the 5th day. Two same objects (E1 and E2) were placed in two different corners of the white box and the mice were trained on the 6th day. The location of E2 was changed once a day after habituation and training (Fig. 5F). The recognition index had no significant difference in habituation (Fig. 5K). The analysis of the discrimination index in following two test trials showed ChK1 overexpression also impaired the discrimination of different locations (Fig. 5L-N). These results confirmed that ChK1 overexpression in astrocytes induced visual episodic memory impairment.

To clarify whether astrocytic ChK1 activation also impair the spatial memory in mice, we used Morris water maze (MWZ) to evaluate the reference spatial learning and memory (Fig. 6A). In the acquisition phase, the escape latency showed no significant difference between Con and ChK1 mice (Fig. 6B). In the probe test after 24 or 48 hours, compared with the control group, the ChK1 mice showed decreased crossing times and time in target quadrant (Fig. 6C-D). These results suggest that ChK1 causes disruption in spatial learning and memory.

Proper function of the hippocampus formation poses a physiological basis of the fear condition memory (FCM). Next, we performed fear conditioning test (FCT) by measuring the freezing time and times after previous training phase when mice were given mild electrical foot shocks followed a conditioned stimulus tone (Fig. $6 \mathrm{H})$. In the fear conditioning test, mice in the ChK1 group pointed out a marked reduction in freezing time and times whether in the contextual or the tone conditioning paradigms (Fig. 61- 
K). Whatsoever, there were no obvious difference in the altered context test (Fig. 6J) between the two groups. Together, these results evidence that ChK1 group mice have conditional fear memory deficits.

\section{Overexpression of ChK1 in astrocytes causes PP2A inhibition, increased inflammatory cytokines and A $\beta$ production, and neuronal and synaptic impairments}

To prove that the molecule signaling pathway of ChK1-CIP2A-PP2A-STAT3 is indeed activated in ChK1 overexpression mice, we evaluated the ChK1 and PP2A activity, and the protein levels of CIP2A and active STAT3 in mouse brain homogenates. ChK1 overexpression brings increased ChK1 activity and decreased PP2A activity (Fig. 7A-B). At the same time, CIP2A and S727-phosphorylated STAT3 levels were upregulated (Fig. 7C-D), indicating the activation of ChK1-CIP2A-PP2A-STAT3 signaling axis in mouse brains. Coinciding with the ChK1-CIP2A-PP2A-STAT3 signaling pathway activation, the astrocytes were activated which is evidenced by the increased GFAP level (Fig. 7C-D), accompanied with increased cytokine and $A \beta$ levels (Fig. 7E-F) in ChK1 overexpression mice.

To identify that ChK1-activated astrocytes promote neurodegeneration in vivo, we used Golgi staining and Nissl staining to show neuron survival. Golgi staining showed that the dendritic spine number of ChK1 group was substantially decreased comparing with the control group (Fig. 7I-J). Moreover, Nissl staining displayed that the number of nissl bodies was decreased in hippocampal CA1 region in the ChK1 group (Fig. 7K-L). Consistent with these findings, synaptic proteins including Syn, Syn I, PSD95, and GluA1 in hippocampus of ChK1 mice were much lower than those in hippocampus of control mice (Fig. 7M-N). Together, ChK1 overexpression in astrocytes give rise to pro-inflammatory cytokines and A $\beta$ production, and impair the synapses and neurons in vivo.

\section{Discussion}

The pathological characters of Alzheimer's disease (AD) include the deposition of senile plaques (SPs),formation of neurofibrillary tangles (NFTs), loss of spines and neurons, as well as abnormal activation of glia. Astrocytes are major glial cells and play important roles in regulating various physiological neuronal functions, including neuronal migration, synaptogenesis, and neuroplasticity [2527]. Astrocytes are also deeply involved in regulating neuronal functions under pathological conditions, including neuroinflammation and oxidative stress [28]. Reactive astrogliosis, which is characterized by increased proliferation and activation of astrocytes, is an early event in $A D$ brain $[29,30]$. PET using ${ }^{11} C$ Deuterium-L-Deprenyl $\left({ }^{11} \mathrm{C}\right.$-DED) showing the astrocytosis revealed increased ${ }^{11} \mathrm{C}$-DED binding throughout the brain of the $\mathrm{MCl}{ }^{11} \mathrm{C}-\mathrm{PIB}+$ patients whose cognition remain normal [31], suggesting that astrogliosis is an early phenomenon in AD development. The activation and proliferation of astrocytes lead to $A \beta$ internalization, inflammatory cytokines production and cognitive dysfunction [19]. Reducing astrocyte activation in an AD mouse model ameliorated AD-like pathology [32]. Thus, astrogliosis is an important part of the AD pathogenesis. However, the mechanisms underlying early astrogliosis in $A D$ remain largely unclarified. 
We have reported the upregulated PP2A endogenous inhibitor CIP2A in astrocytes in AD mice brain resulted in astrogliosis, AD-like neurodegeneration and cognitive dysfunction [13] While in cancer cells, DNA damage response kinase ChK1 upregulates CIP2A level and inhibits PP2A activation [33]. In the present study, we link these two events together and reveal that ChK1 activation upregulates CIP2A and induces astrogliosis, the latter, promotes neuronal and synaptic degeneration, and cognitive impairment.

STAT3 is a signal transducer associated transcription factor and activation of STAT3 regulates reactive astrogliosis [34], induces GFAP [35] expression and production of pro-inflammatory factors such as TNFa and IL-6 $[36,37]$. These data suggest that STAT3 may play a crucial part in astrogliosis. However, the molecular mechanism of STAT3 activation in astrocyte is not clear. In tumor cells, PP2A inactivation increases phosphorylation levels of STAT3-S727, activates STAT3 and leads to proliferation of tumor cells $[16,18]$. Again, we linked these two events (PP2A inhibition and STAT3 activation) together and disclosed a new mechanism underlying astrogliosis in AD.

According to our results, in the brains of 3XTg-AD mice, $\mathrm{YH} 2 \mathrm{~A} . \mathrm{X}$, ChK1-S345 (active form of ChK1) and CIP2A expression levels are much higher than that in the WT mice. Similarly, in A -treated primary astrocytes, we also found the DNA damage, ChK1 activation, CIP2A upregulation and PP2A inhibition. Together with these changes, astrocytes were activated. Further detection indicated that STAT3 phosphorylation at S727 and activation was involved in this process. After applying ChK1 inhibitor SB, changes in CIP2A, PP2A, S727 phosphorylated STAT3, and GFAP were all reversed or partially reversed, suggesting a signaling pathway as ChK1-CIP2A-PP2A-STAT3 participates in astrogliosis in AD brain.

To further confirm this hypothesis, we overexpressed ChK1 to directly activate this kinase in primary astrocytes. Consistent with the previous findings, ChK1 activation through overexpression resulted in CIP2A upregulation, STAT3 activation and astrogliosis, which could be reversed by ChK1 inhibitor. Accompanied with astrocytes activation, cytokine and $A \beta$ levels in the media were increased, and neurons treated with the astrocyte-conditioned media showed degeneration characterized by decreased synaptic protein levels and impaired neurite morphology. Taking together, these findings imply that ChK1 activation in astrocytes can induce astrogliosis through ChK1-CIP2A-PP2A-STAT3 signaling pathway and promote neurodegeneration.

We have showed that specific overexpression of CIP2A in astrocytes in mouse brains induce neurodegeneration and cognitive impairments [13]. ChK1 activation promotes CIP2A overexpression and cell proliferation in cancer cells [33]. Based on the finding that ChK1 is also upstream of CIP2A in astrocyte activation, we overexpressed ChK1 specifically in astrocytes in mouse brain to observe whether ChK1 activation promote astrocyte activation and neurodegeneration in vivo. The results were consistent with the data obtained in cell experiments. ChK1 overexpression resulted in CIP2A upregulation, PP2A inhibition and STAT3 activation. Coincided, increased cytokine and A $\beta$, synaptic and neuronal degeneration were observed. Animals showed learning and memory deficits in several behavior tests.

These data precisely identified the role of ChK1 activation in promoting astrogliosis in AD. In a parallel study, we found that activation of ChK1 in neurons also lead to AD-like tau hyperphosphorylation, $A B$ 
overproduction, neurodegeneration and cognitive impairment (unpublished). Taking together, ChK1 is a promising drug target in AD therapy.

All in all, we demonstrate, for the first time, that ChK1 activation by DNA damage or ChK1 overexpression in astrocyte leads to CIP2A upregulation, PP2A inactivation and STAT3 activation, then induces reactive astrogliosis, resulting in synaptic impairments and neurodegeneration in AD.

\section{Abbreviations}

ChK1: Checkpoint kinase 1; CIP2A: Cellular inhibitor of PP2A; PP2A: Protein phosphatase 2A; STAT3: Signal transducer and activator of transcription 3; AD: Alzheimer's disease; GFAP: Glial fibrillary acidic protein; AAV: Adeno-associated virus; 3XTg-AD: triple-transgenic model of AD; $A \beta$ : $\beta$-Amyloid; Fig: Figure; Fig. S: Figure supplement; GAPDH: Glyceraldehyde-3-phosphate dehydrogenase; SB: SB281078; ACM: Astrocyte Conditioned Medium; DIV: Day in vitro; IL-6: Interleukin- 6; TNF-a: Tumor necrosis factor-a; EGFP: Enhanced green fluorescent protein; Syn: Synaptophysin; Syn I: Synapsin I; PSD95: Postsynaptic Density protein 95; GluA1: AMPA Receptor 1; MAP-2: Microtubule-associated protein-2; ORM: Object location memory; NORT: Novel object recognition test; OLT: Object location test; MWZ: Morris water maze; Con: Control;FCM: Fear condition memory; FCT: Fear conditioning test; NFTs: Neurofibrillary tangles; PET: Positron emission tomography; 11C-DED: 11C-Deuterium-L-Deprenyl; ITI: Intertrial interval.

\section{Declarations}

\section{Acknowledgements}

We thank Jukka Westermarck for critical of reading of the manuscript. The authors thank the members of the Shentu and Liu labs for helpful comments on the manuscript.

\section{Authors' contributions}

Y.Z., X.Y.L. and S.Q.M. performed most of the experiments, including PP2A activity assay, ChK1 activity assay, ELISA, Golgi staining, Nissl staining, cell culture and cell experiments. They also wrote the first manuscript. D.C.Y., S.M.Y., S.Y. W. and J.N. performed behavioral tests (OPF, NOR, FCT and MWM). Y.P. S.T. and Y.Z. analyzed data. Y.Z. and X.Y.L. injected AAV into the lateral ventricles of mice. Q.Y.Z., J.R., J.M. and S.J.Z. collected and sectioned mice brain samples. S.Q.M. and Z.Y.Z. provided assistances for the primary neuron culture and its Immunofluorescence. Z.C.F, X.F.F., Y.S.G., J.M.F. and X.C.W. provided oversight and technical support. J.M.L., R.L. and Y.P.S.T. designed experiments, secured funding, directed the project, reviewed experimental results and edited the manuscript.

\section{Funding}

This work was supported by the National Natural Science Foundation of China (grant 31900685 to Y.P.S.T., grants 31771189 and 31970964 to R.L.). This work was also supported by the Natural Science 
Foundation of Zhejiang Province of China (grant LQ19H050002 to Y.Z.). This work was also supported by the Wenzhou Municipal Science and Technology Bureau of China (grant Y20210079 to Y.P.S.T., grants ZS2017008 and Y20180159 to Y.Z.).

\section{Availability of data and materials}

All data associated with this study are present in the paper and AAV was constructed by Shanghai Genechem Co. (Shanghai, China).

\section{Ethics approval and consent to participate}

All experimental procedures were approved by the Ethics Committee for Experimental Animal Use and Care of Wenzhou Medical University.

\section{Consent for publication}

Not applicable

\section{Competing interests}

The authors declare that they have no competing interests.

\section{References}

1. Pooler AM, Noble W, Hanger DP. A role for tau at the synapse in Alzheimer's disease pathogenesis. Neuropharmacology. 2014;76(Pt A):1-8.

2. Tam JH, Pasternak SH. Amyloid and Alzheimer's disease: inside and out. Can J Neurol Sci. 2012;39(3):286-98.

3. Valles SL, Iradi A, Aldasoro M, Vila JM, Aldasoro C, de la Torre J, et al. Function of Glia in Aging and the Brain Diseases. Int J Med Sci. 2019;16(11):1473-9.

4. Zhou S, Szczesna K, Ochalek A, Kobolák J, Varga E, Nemes C, et al. Neurosphere Based Differentiation of Human iPSC Improves Astrocyte Differentiation. Stem Cells Int. 2016;2016:4937689.

5. Hong S, Beja-Glasser VF, Nfonoyim BM, Frouin A, Li S, Ramakrishnan S, et al. Complement and microglia mediate early synapse loss in Alzheimer mouse models. Science. 2016;352(6286):712-6.

6. Sosna J, Philipp S, Albay R 3rd, Reyes-Ruiz JM, Baglietto-Vargas D, LaFerla FM, et al. Early long-term administration of the CSF1R inhibitor PLX3397 ablates microglia and reduces accumulation of intraneuronal amyloid, neuritic plaque deposition and pre-fibrillar oligomers in 5XFAD mouse model of Alzheimer's disease. Mol Neurodegener. 2018;13(1):11.

7. Junttila MR, Puustinen P, Niemelä M, Ahola R, Arnold H, Böttzauw T, et al. CIP2A inhibits PP2A in human malignancies. Cell. 2007;130(1):51-62. 
8. O'Connor CM, Perl A, Leonard D, Sangodkar J, Narla G. Therapeutic targeting of PP2A. Int J Biochem Cell Biol. 2018;96:182-93.

9. Fujiki H, Sueoka E, Watanabe T, Suganuma M. The concept of the okadaic acid class of tumor promoters is revived in endogenous protein inhibitors of protein phosphatase 2A, SET and CIP2A, in human cancers. J Cancer Res Clin Oncol. 2018;144(12):2339-49.

10. Gong CX, Singh TJ, Grundke-lqbal I, Iqbal K. Phosphoprotein phosphatase activities in Alzheimer disease brain. J Neurochem. 1993;61(3):921-7.

11. Gong CX, Shaikh S, Wang JZ, Zaidi T, Grundke-lqbal I, Iqbal K. Phosphatase activity toward abnormally phosphorylated tau: decrease in Alzheimer disease brain. J Neurochem. 1995;65(2):7328.

12. Shentu YP, Huo Y, Feng XL, Gilbert J, Zhang Q, Liuyang ZY, et al. CIP2A Causes Tau/APP Phosphorylation, Synaptopathy, and Memory Deficits in Alzheimer's Disease. Cell Rep. 2018;24(3):713-23.

13. Shentu YP, Hu WT, Zhang Q, Huo Y, Liang JW, Liuyang ZY, et al. CIP2A-promoted astrogliosis induces AD-like synaptic degeneration and cognitive deficits. Neurobiol Aging. 2019;75:198-208.

14. Patil M, Pabla N, Dong Z. Checkpoint kinase 1 in DNA damage response and cell cycle regulation. Cell Mol Life Sci. 2013;70(21):4009-21.

15. Tamma R, Ingravallo G, Albano F, Gaudio F, Annese T, Ruggieri S, et al. STAT-3 RNAscope Determination in Human Diffuse Large B-Cell Lymphoma. Transl Oncol. 2019;12(3):545-9.

16. Togi S, Kamitani S, Kawakami S, Ikeda O, Muromoto R, Nanbo A, et al. HDAC3 influences phosphorylation of STAT3 at serine 727 by interacting with PP2A. Biochem Biophys Res Commun. 2009;379(2):616-20.

17. Liu CC, Lin SP, Hsu HS, Yang SH, Lin CH, Yang MH, et al. Suspension survival mediated by PP2ASTAT3-Col XVII determines tumour initiation and metastasis in cancer stem cells. Nat Commun. 2016 Jun 16;7:11798. doi: 10.1038/ncomms11798. Erratum in: Nat Commun. 2016;7:14027.

18. Chen Z, Ji N, Wang Z, Wu C, Sun Z, Li Y, et al. Fine Particulate Matter (PM2.5) Promoted the Invasion of Lung Cancer Cells via an ARNT2/PP2A/STAT3/MMP2 Pathway. J Biomed Nanotechnol. 2018;14(12):2172-84.

19. Reichenbach N, Delekate A, Plescher M, Schmitt F, Krauss S, Blank N, et al. Inhibition of Stat3mediated astrogliosis ameliorates pathology in an Alzheimer's disease model. EMBO Mol Med. 2019;11(2):e9665.

20. Qiu M, Shentu YP, Zeng J, Wang XC, Yan X, Zhou XW, et al. Zinc mediates the neuronal activitydependent anti-apoptotic effect. PLoS One. 2017;12(8):e0182150.

21. Michalska P, León R. When It Comes to an End: Oxidative Stress Crosstalk with Protein Aggregation and Neuroinflammation Induce Neurodegeneration. Antioxidants (Basel). 2020;9(8):740.

22. Gruol DL. Impact of Increased Astrocyte Expression of IL-6, CCL2 or CXCL10 in Transgenic Mice on Hippocampal Synaptic Function. Brain Sci. 2016;6(2):19. 
23. Frost GR, Li YM. The role of astrocytes in amyloid production and Alzheimer's disease. Open Biol. 2017;7(12):170228.

24. Minter MR, Taylor JM, Crack PJ. The contribution of neuroinflammation to amyloid toxicity in Alzheimer's disease. J Neurochem. 2016;136(3):457-74.

25. Aarum J, Sandberg K, Haeberlein SL, Persson MA. Migration and differentiation of neural precursor cells can be directed by microglia. Proc Natl Acad Sci U S A. 2003;100(26):15983-8.

26. Bilbo SD, Schwarz JM. Early-life programming of later-life brain and behavior: a critical role for the immune system. Front Behav Neurosci. 2009;3:14.

27. Aarum J, Sandberg K, Haeberlein SL, Persson MA. Migration and differentiation of neural precursor cells can be directed by microglia. Proc Natl Acad Sci U S A. 2003;100(26):15983-8.

28. Agostinho P, Cunha RA, Oliveira C. Neuroinflammation, oxidative stress and the pathogenesis of Alzheimer's disease. Curr Pharm Des. 2010;16(25):2766-78.

29. Munger EL, Edler MK, Hopkins WD, Ely JJ, Erwin JM, Perl DP, et al. Astrocytic changes with aging and Alzheimer's disease-type pathology in chimpanzees. J Comp Neurol. 2019;527(7):1179-95.

30. Johnson ECB, Dammer EB, Duong DM, Ping L, Zhou M, Yin L, et al. Large-scale proteomic analysis of Alzheimer's disease brain and cerebrospinal fluid reveals early changes in energy metabolism associated with microglia and astrocyte activation. Nat Med. 2020;26(5):769-80.

31. Carter SF, Schöll M, Almkvist O, Wall A, Engler H, Långström B, et al. Evidence for astrocytosis in prodromal Alzheimer disease provided by 11C-deuterium-L-deprenyl: a multitracer PET paradigm combining 11C-Pittsburgh compound B and 18F-FDG. J Nucl Med. 2012;53(1):37-46.

32. Furman JL, Sama DM, Gant JC, Beckett TL, Murphy MP, Bachstetter AD, et al. Targeting astrocytes ameliorates neurologic changes in a mouse model of Alzheimer's disease. J Neurosci. 2012;32(46):16129-40.

33. Khanna A, Kauko O, Böckelman C, Laine A, Schreck I, Partanen Jl, et al. Chk1 targeting reactivates PP2A tumor suppressor activity in cancer cells. Cancer Res. 2013;73(22):6757-69.

34. Toral-Rios D, Patiño-López G, Gómez-Lira G, Gutiérrez R, Becerril-Pérez F, Rosales-Córdova A, et al. Activation of STAT3 Regulates Reactive Astrogliosis and Neuronal Death Induced by A 30 Neurotoxicity. Int J Mol Sci. 2020;21(20):7458.

35. Hung CC, Lee YH, Kuo YM, Hsu PC, Tsay HJ, Hsu YT, et al. Soluble epoxide hydrolase modulates immune responses in activated astrocytes involving regulation of STAT3 activity. $\mathrm{J}$ Neuroinflammation. 2019;16(1):123.

36. O'Callaghan JP, Kelly KA, VanGilder RL, Sofroniew MV, Miller DB. Early activation of STAT3 regulates reactive astrogliosis induced by diverse forms of neurotoxicity. PLoS One. 2014;9(7):e102003.

37. Beurel E, Jope RS. Lipopolysaccharide-induced interleukin-6 production is controlled by glycogen synthase kinase-3 and STAT3 in the brain. J Neuroinflammation. 2009;6:9.

\section{Figures}




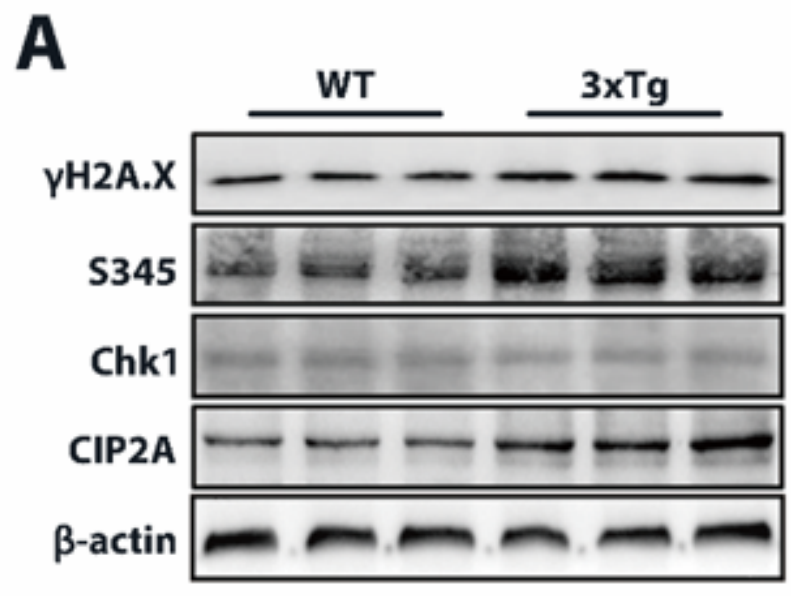

B
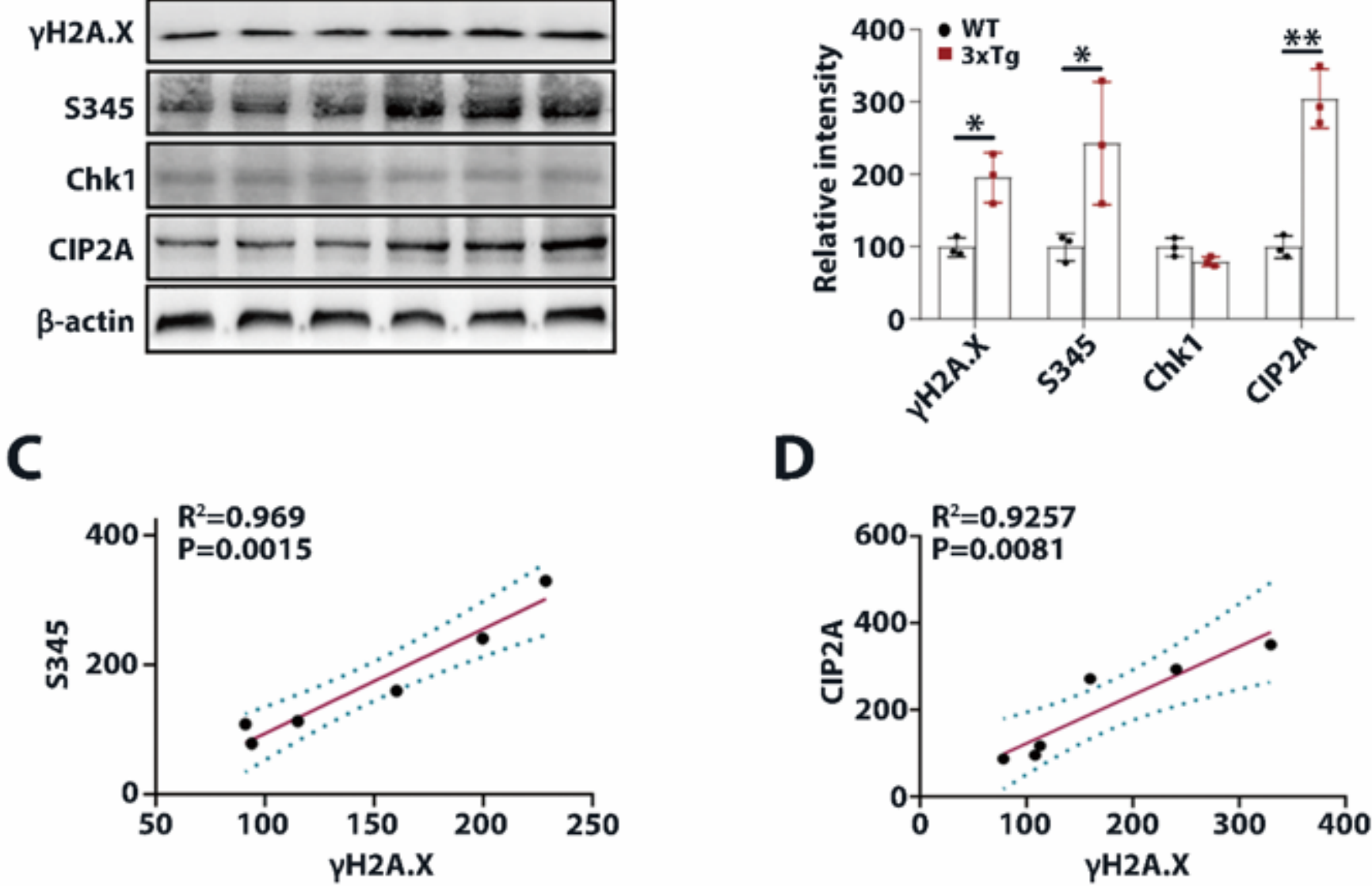

D

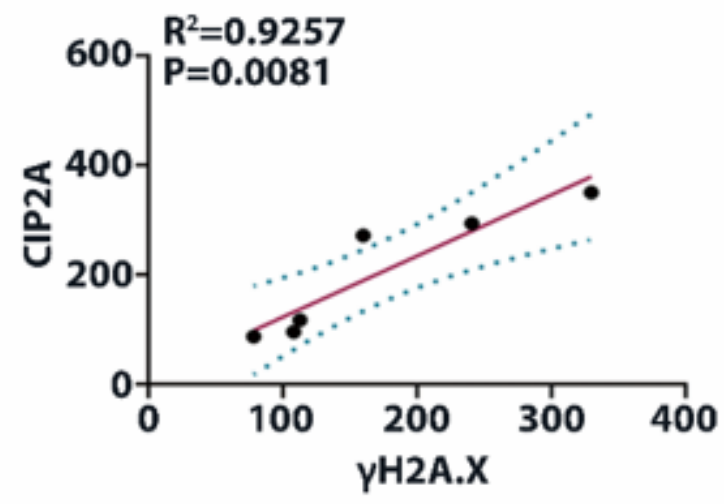

Figure 1

Increased DNA damage, ChK1 activation and CIP2A upregulation in 3xTg-AD mouse brains. A Representative immunoblots of $\mathrm{YH} 2 \mathrm{~A}$.X, active ChK1-S345, total ChK1, CIP2A, and $\beta$-actin in wild-type and 3xTg-AD mouse brains (hippocampal tissues, 9 months old, $n=3$ mice per group). B Quantitative analysis of the proteins level in (A). C and D Correlation analysis between ChK1-S345 and $\mathrm{YH} 2 \mathrm{~A} . \mathrm{X}, \mathrm{CIP} 2 \mathrm{~A}$ and ChK1-S345. ${ }^{*} P<0.05,{ }^{*} \mathrm{P}<0.01$, Data are mean \pm SEM. 

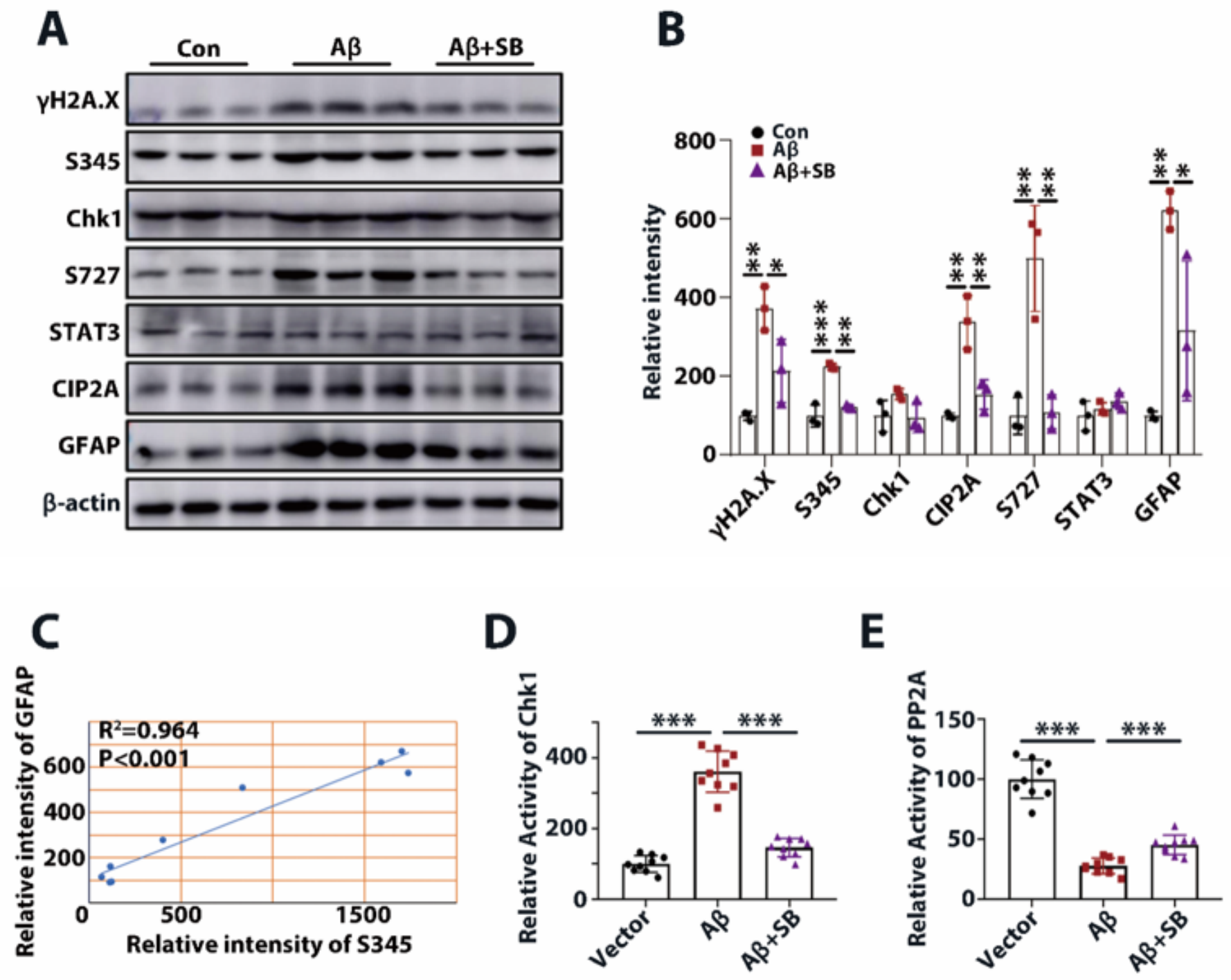

Figure 2

$A \beta$ induces DNA damage, ChK1-CIP2A-PP2A-STAT3 signaling pathway activation and astrogliosis in primary astrocytes, which can be reversed by ChK1 inhibitor SB. A Primary astrocytes were incubated with $A \beta$ oligomers $(1 \mu \mathrm{M})$, with or without treatment of ChK1 inhibitor SB for 48 hours. Representative immunoblots of $\mathrm{YH} 2 \mathrm{~A}$.X囚active ChK1-S345, total ChK1, active STAT3-S727, total STAT3, CIP2A, GFAP and $\beta$-actin in Con, $A \beta$ and $A \beta+S B$ groups. $B$ Quantitative analysis of the proteins levels in $(A), n=3$ per group. C Linear correlation between ChK1-S345 and GFAP. D and E ChK1 and PP2A activities in these three groups. $n=6$ per group, ${ }^{\star} P<0.05,{ }^{\star} \mathrm{P}<0.01$, ${ }^{\star \star \star} \mathrm{P}<0.001$, Data are mean $\pm S E M$. 
A
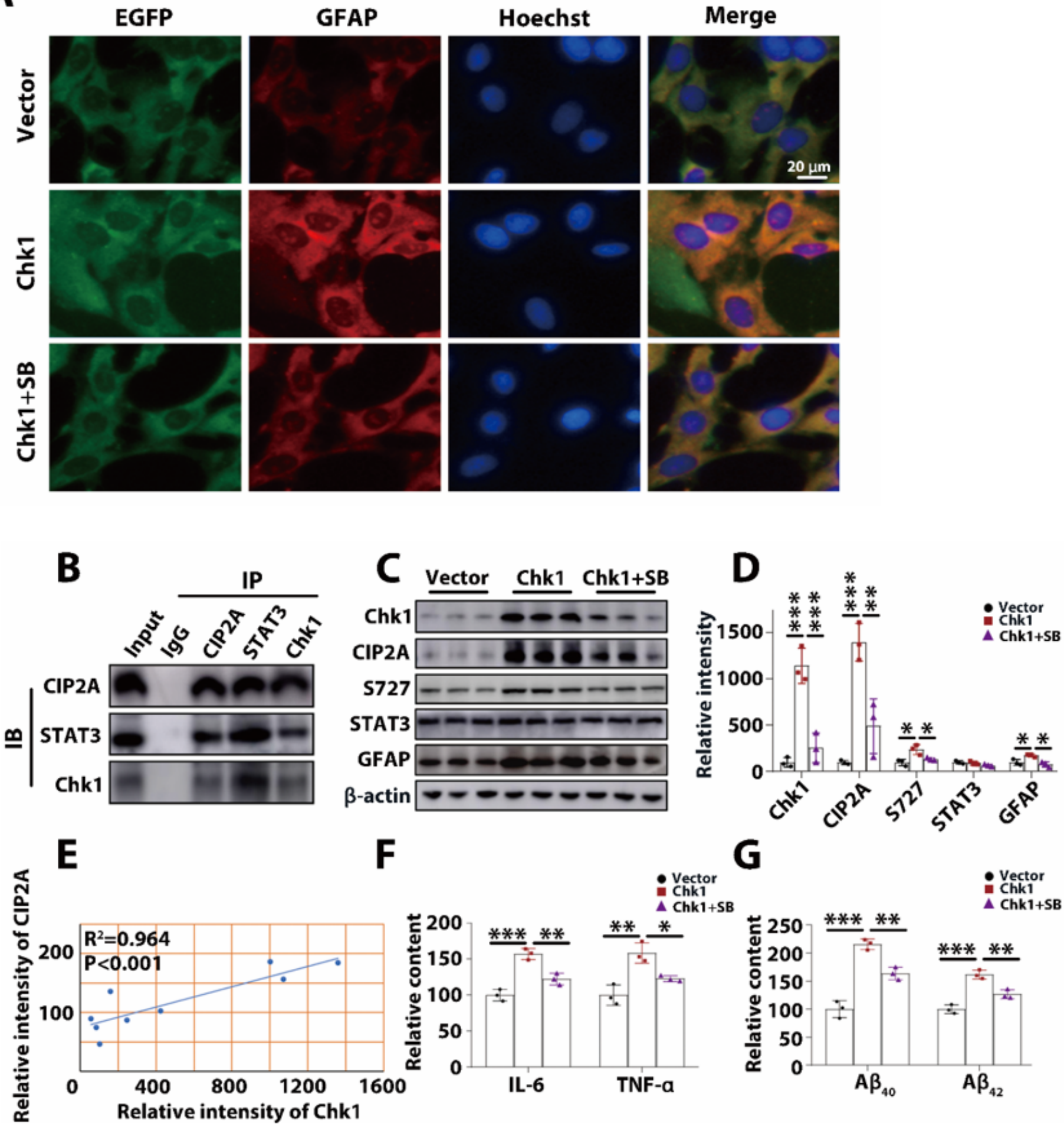

Figure 3

Overexpression of ChK1 induces CIP2A-PP2A-STAT3 pathway activation and reactive astrogliosis in primary astrocytes, which can be reversed by ChK1 inhibitor. A The primary astrocytes (DIV10) were infected with Lenti-virus with GFAP promoter, with or without treatment of SB. EGFP (green), immunofluorescence staining of GFAP (red), and Hoechst fluorescence (blue) in Vector, ChK1, ChK1+SB groups. Scale bar: $20 \mu \mathrm{m}$. B The interaction of CIP2A, STAT3 and ChK1 was examined by Co-IP assay. C 
Representative immunoblots of total ChK1, CIP2A, active STAT3-S727, total STAT3, GFAP, and $\beta$-actin in Vector, ChK1, and ChK1+SB groups. D Quantitative analysis of the proteins levels in (C). E Linear correlation between CIP2A and ChK1. F and G Quantitative analysis of IL-6, TNF- $a, A \beta 40$ and AB42 in Vector, ChK1, ChK1+SB groups. $n=3$ per group, ${ }^{*} P<0.05,{ }^{*} P<<0.01, * * * P \llbracket 0.001$, Data are mean $\pm S E M$.
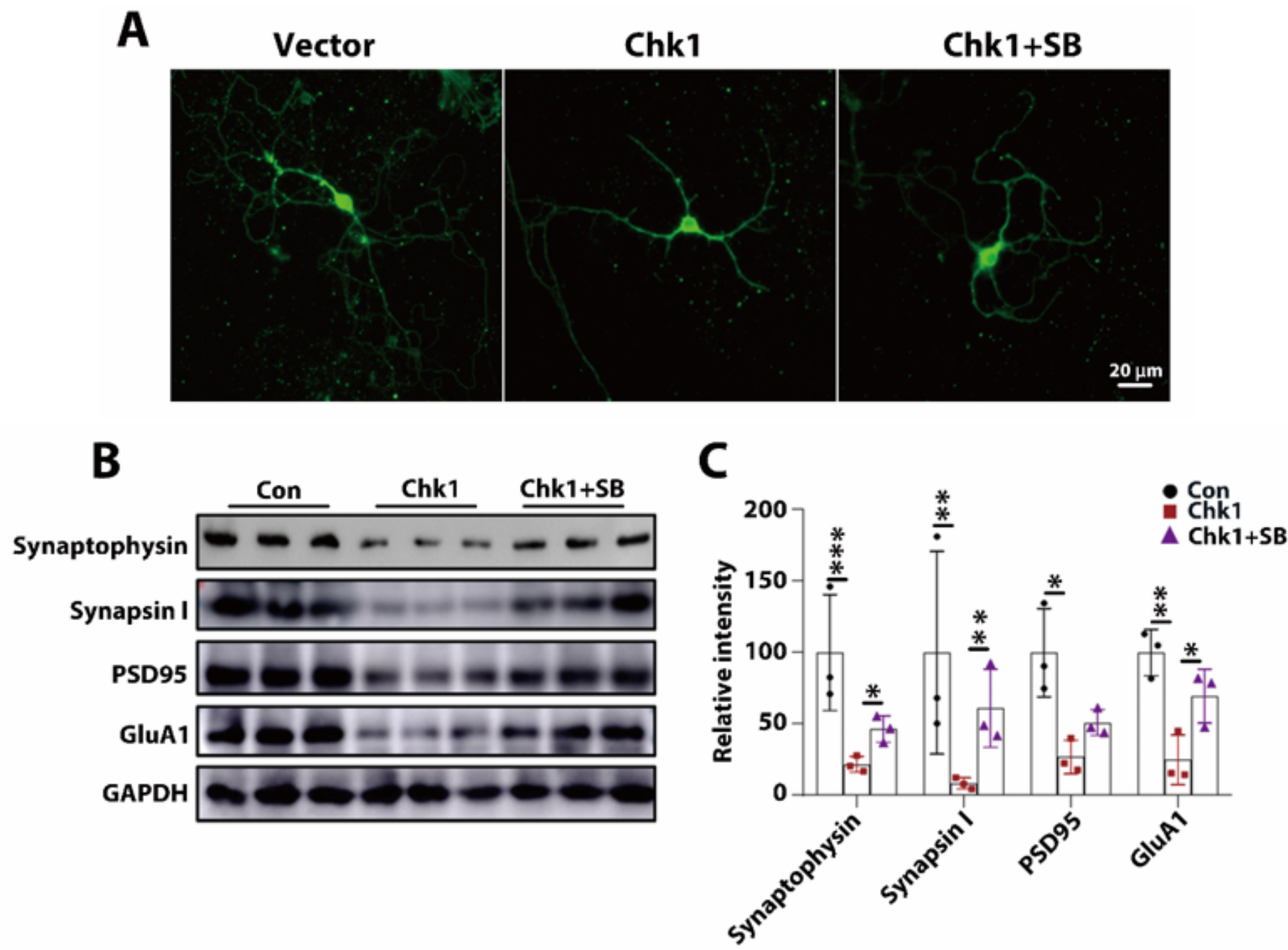

Figure 4

Conditioned medium from ChK1-overexpressed astrocytes induces neurodegeneration. A The rat primary neurons (DIV 8) were incubated with conditioned medium from ChK1-overexpressed astrocytes (ChK1ACM) or ChK1+SB-treated astrocytes (ChK1+SB-ACM) for 4 days. Immunofluorescence staining with MAP-2 in the Vector, ChK1 and ChK1+SB groups. B Representative immunoblots of Synaptophysin, Synapsin I, PSD95, GluA1 and GAPDH in the Vector, ChK1 and ChK1+SB groups. C Quantitative analysis of the proteins levels in (B). $n=3$ per group, ${ }^{\star} P<0.05,{ }^{\star} \mathrm{P}<0.01,{ }^{* \star *} \mathrm{P} \otimes 0.001$, Data are mean \pm SEM. 
A

\section{$A A V$-Injection 1}

2 NORT/OLT

MWM 3

4

Postmortem 5 Analysis

E

Novel Object Recognition Test (NORT)

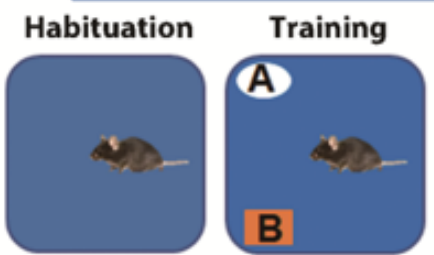

Day1

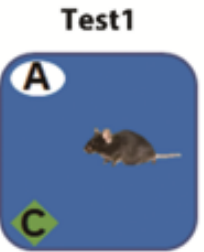

Day3

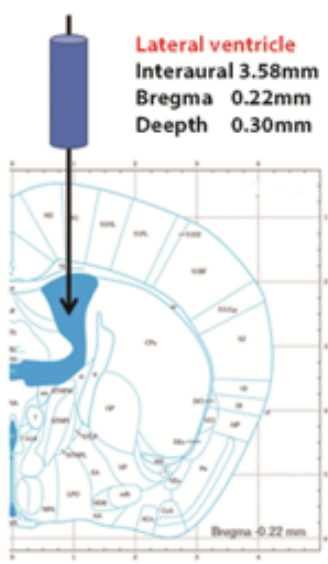

D

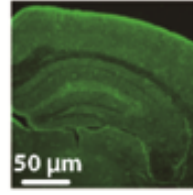

GFAP mCherry Hoechst Merge

Promoter (pAAV-MCS)

$\downarrow$

GFAP
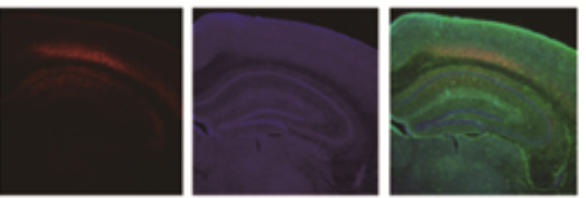

mCherry

$\mathbf{F}$

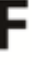

Day4

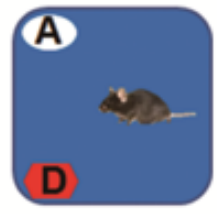

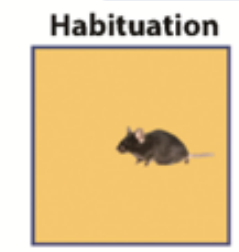

Day5
Object Location Test (OLT)

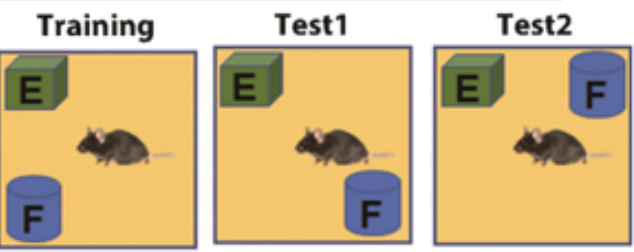

Day6

Day7

Day8

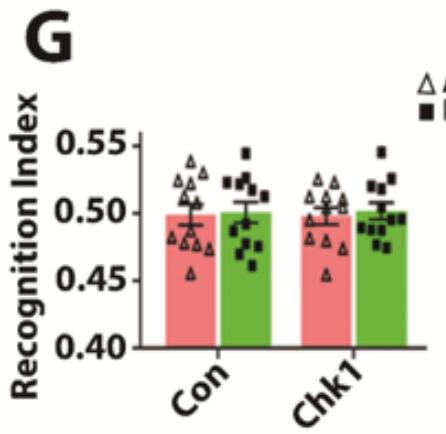

H
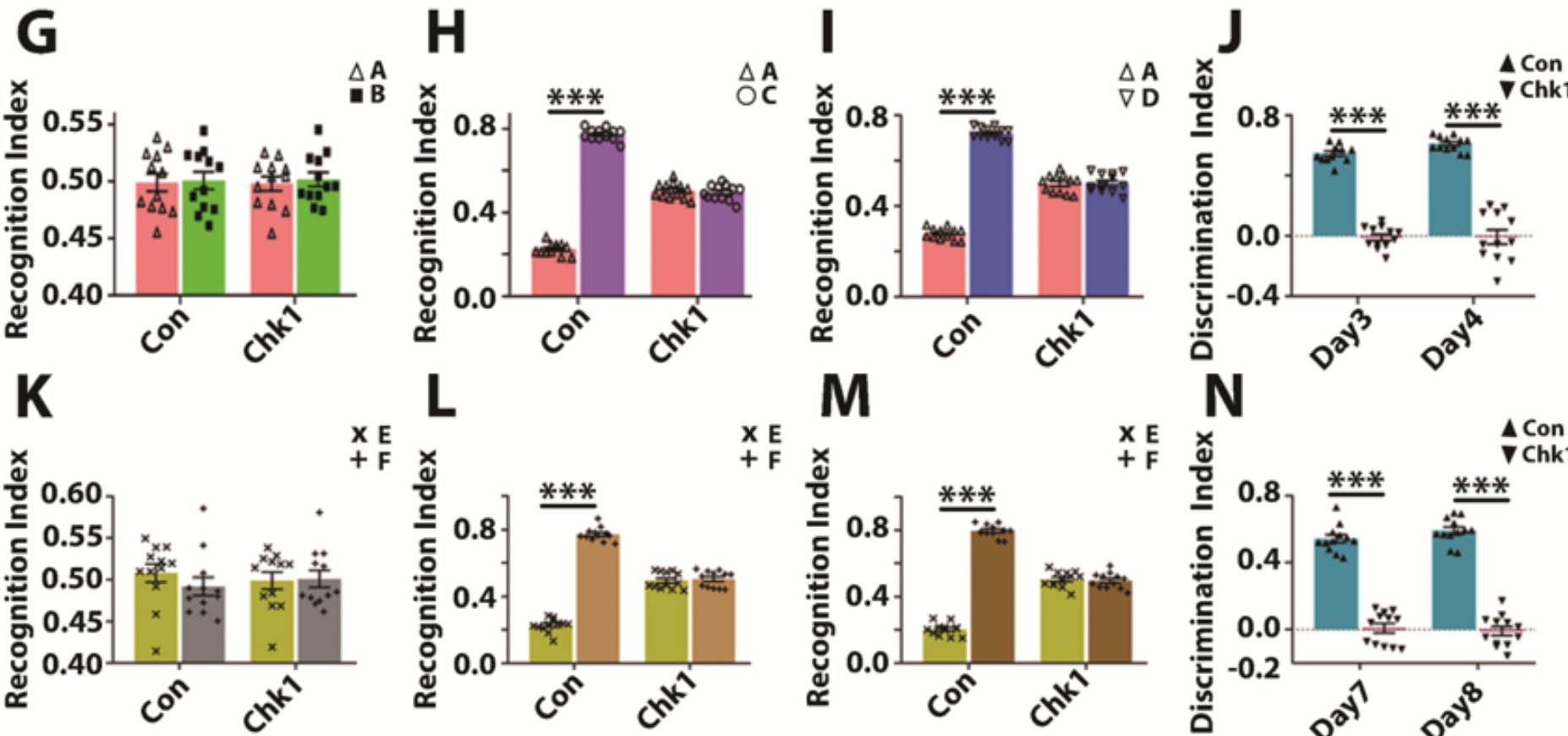

\section{$M$}
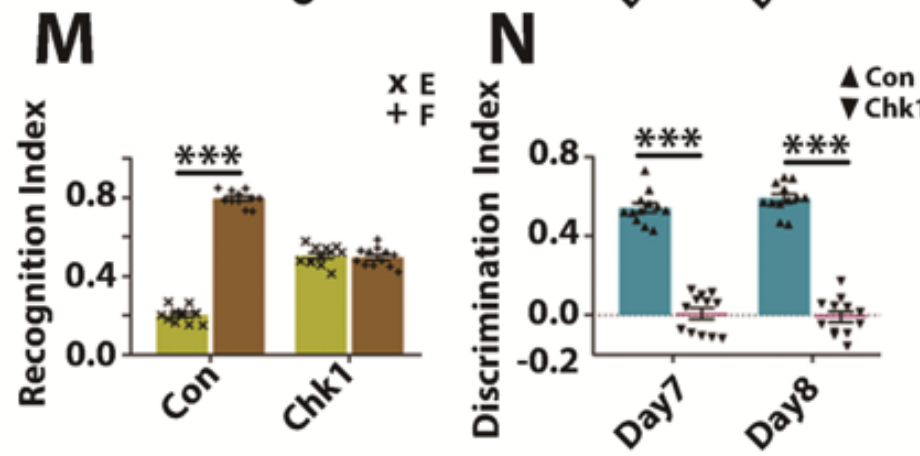

Figure 5

Overexpression of ChK1 in astrocytes induces deficits in novel object recognition test (NORT) and object location test (OLT) in C57BL/6J mice. A Timeline of the experiment. B GFAP-ChK1-AAV was injected into the lateral ventricle. C The structure diagram of GFAP-ChK1-AAV. D Immunofluorescence staining of brain slices four weeks after virus infection. $E$ and $F$ The schematic diagram of the novel object recognition test (NORT) and object location test (OLT) tests. G Recognition index for object $A$ and $B$ in the acquisition trial 
on Day 2. $\mathrm{H}$ Object $B$ was replaced by a new object $C$, and recognition index for object $A$ and $C$ was detected on Day 3. I Object $C$ was replaced by a new object $D$, and recognition index for object $A$ and $D$ was detected on Day 4. J Discrimination index in Object Recognition Memory test on Day 3 and Day 4. K Recognition index for the objects in place $E$ and $F$ in the acquisition trial on Day 6 . $L$ The position $E 2$ were changed, and recognition index for the objects at place $E$ and $F$ were detected on Day 7. M The position of E2 were changed again, and recognition index for the objects at place $E$ and F were detected on Day 8. $\mathrm{N}$ Discrimination index in Object Location Memory test on Day 7 and Day 8. $\mathrm{N}=12$ mice/group, ${ }^{* \star * P Q}$ $0.001 \otimes \mathrm{D}$ ata are mean \pm SEM. 
A
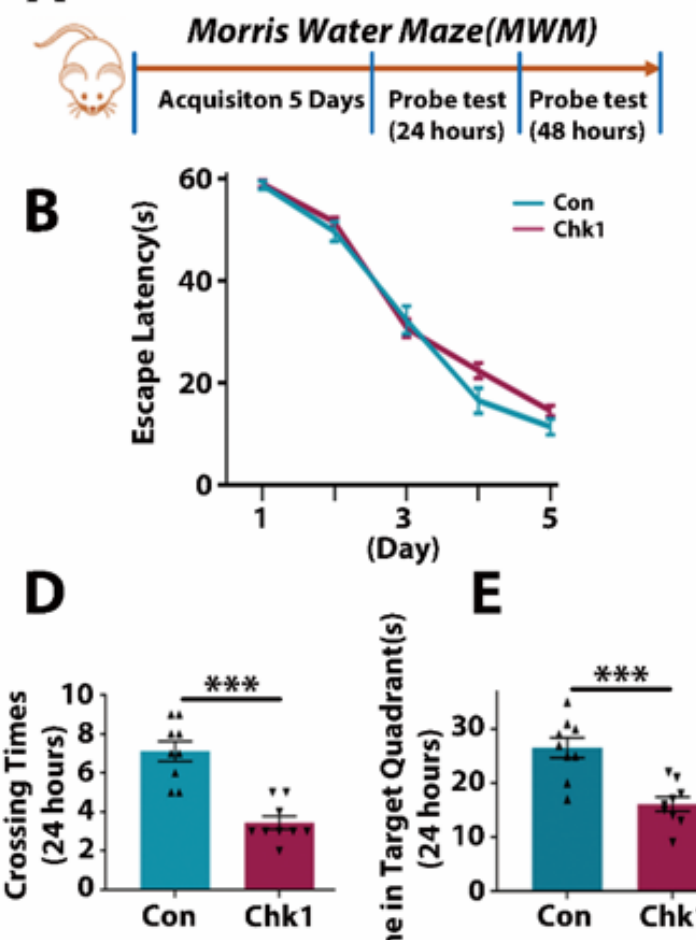

E

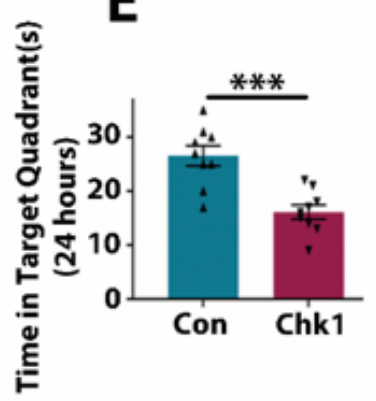

\section{Searching trace after removing the platform}

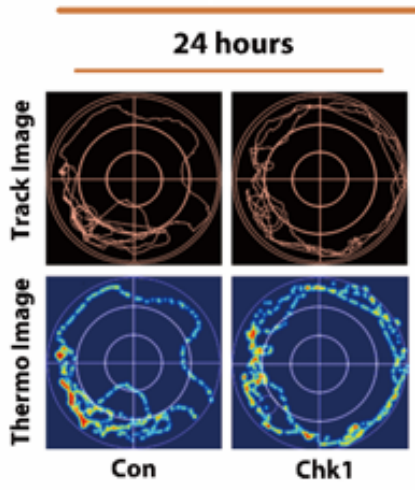

$\mathbf{F}$

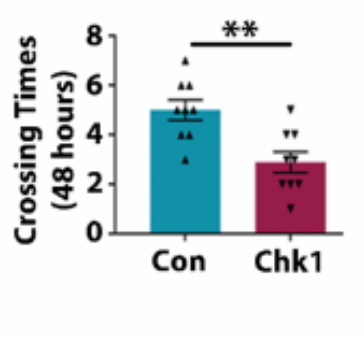

48 hours

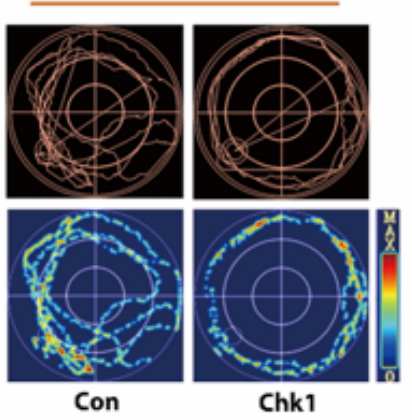

$\mathbf{G}$

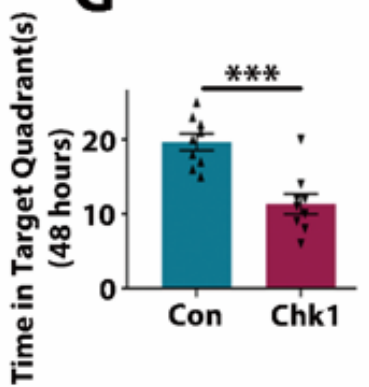

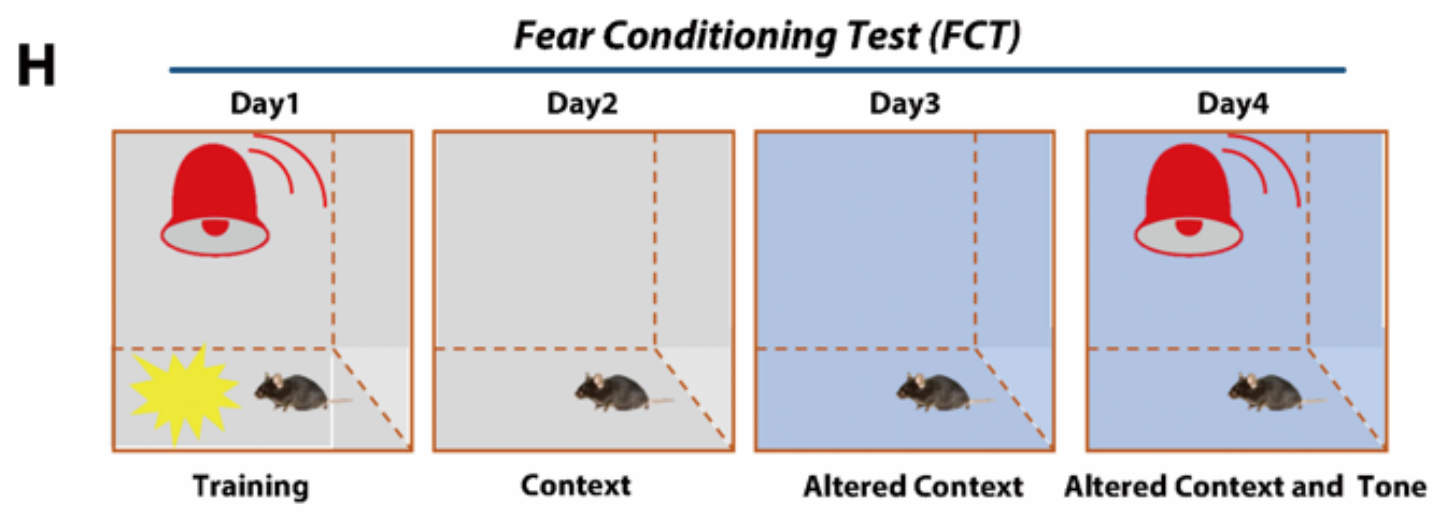

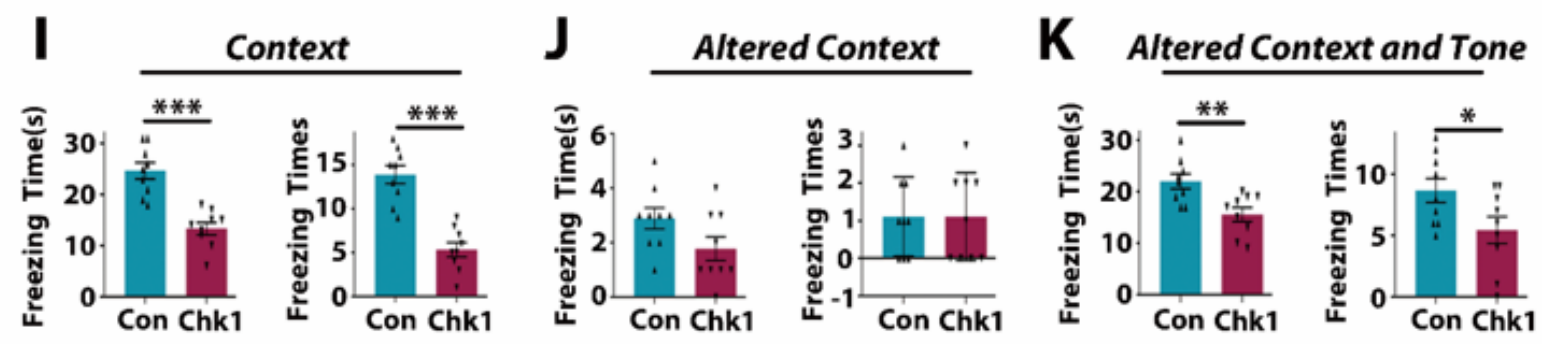

Figure 6

Overexpression of ChK1 in astrocytes induces deficits in the reference spatial memory and fear memory in C57BL/6J mice. A Experimental design of Morris Water Maze (MWM). B The escape latency of mice during 5-day training. $C$ The representative traces during the $24 \mathrm{~h}$ and $48 \mathrm{~h}$ probe test in which the platform was removed. D Times of crossing the position of the platform after removing the platform during the 24-hour probe trial. E Time of swimming in target quadrant after removing the platform during 
the 24-hour probe trial. F Times of crossing the position of the platform after removing the platform during the 48-hour probe trial. G Time of swimming in target quadrant after removing the platform during the 48-hour probe trial. $\mathrm{H}$ The schematic diagram of fear conditioning test (FCT). I The freezing time and freezing times of the mice in the Context test (Day 2). J The freezing time and freezing times of the mice in the Altered Context test (Day 3). K The freezing time and freezing times of the mice in the Altered Context test and tone test (Day 4). $n=9$ mice/group, ${ }^{*} P<0.05,{ }^{\star *} \mathrm{P}<0.01,{ }^{\star \star \star} \mathrm{P} \otimes 0.001$, Data are mean \pm SEM.
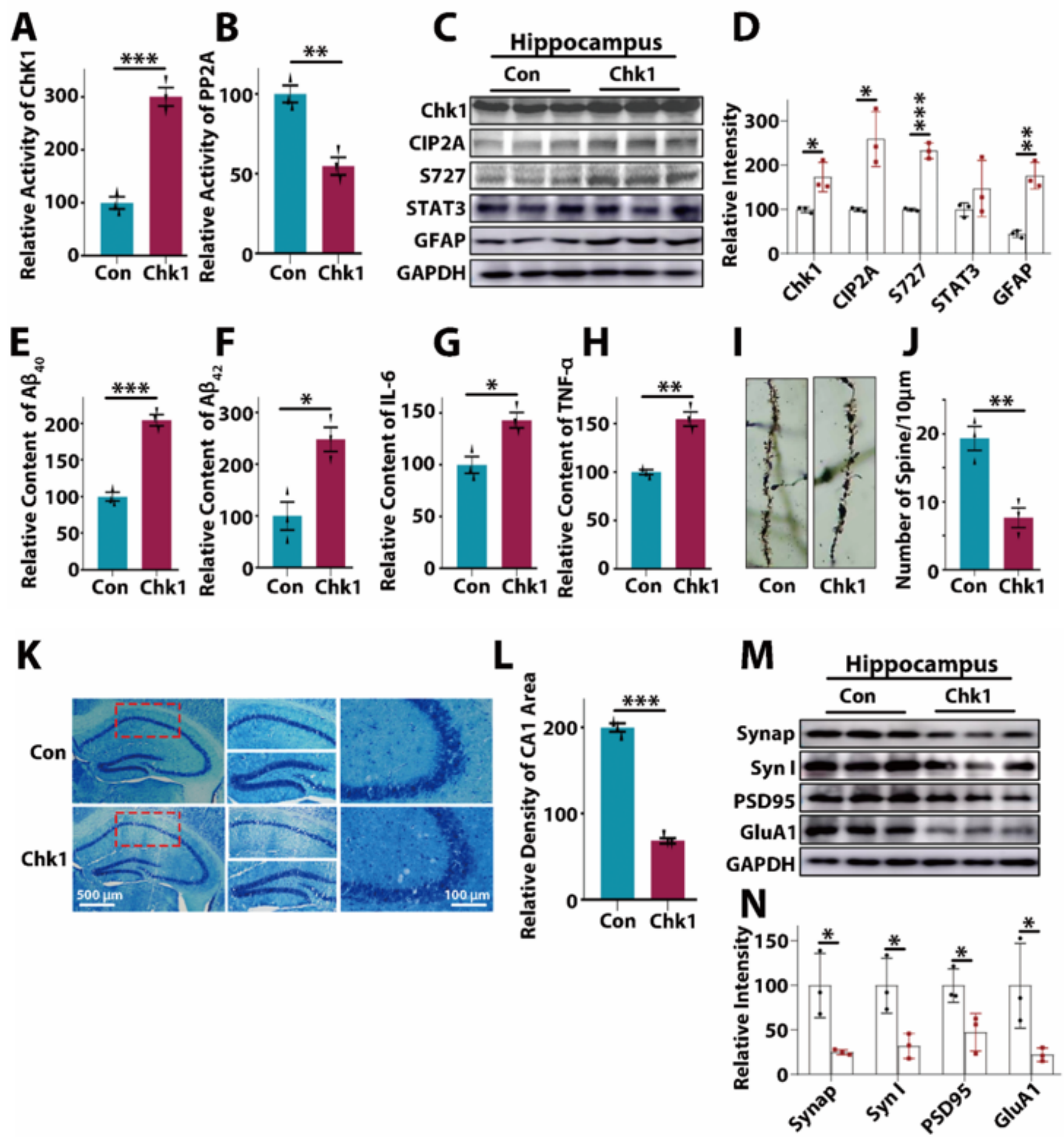


\section{Figure 7}

Overexpression of ChK1 in astrocytes causes ChK1-CIP2A-PP2A-STAT3 signaling axis activation, astrogliosis, $A D$-like $A \beta$ overproduction, inflammatory factors release and neurodegeneration in $C 57 \mathrm{BL} / 6 \mathrm{~J}$ mice. A and B The brain hippocampal tissues of the mice were collected and homogenized for ChK1 and PP2A activity assay. C Representative immunoblots of ChK1, CIP2A, S727-STAT3, total STAT3, GFAP and GAPDH in the hippocampus of the Con and ChK1 groups, GAPDH was used as a loading control. D Quantitative analysis of the proteins level in (C). E and $\mathrm{F}$ The brain homogenates of hippocampus were used for ELISA assay of $A \beta 40$ and $A \beta 42 . G$ and $H$ The brain homogenates of hippocampus were used for ELISA assay of IL- 6 and TNF-a. I Typical images of neuronal spines in hippocampal neurons. J Quantitative analysis of neuronal spines in CA1 region of (I). K Representative Nissl staining images of brain slices. $L$ Quantitative analysis of Nissl bodies in CA1 region of $(K)$. M Representative immunoblots of Syn, Syn I, PSD95, GluA1 and GAPDH in the hippocampus of the Con and ChK1 groups, GAPDH was used as a loading control. N Quantitative analysis of the proteins level in $(M) . n=3$ per group, ${ }^{*} P<0.05$, $\star * P<0.01, * \star * P \otimes 0.001$, Data are mean \pm SEM.

\section{Supplementary Files}

This is a list of supplementary files associated with this preprint. Click to download.

- 20210905SupplementaryMaterials.docx 